\title{
Trabalhos de conclusão Como a temperatura afeta a dinâmica populacional de Tenuipalpus heveae (Acari, Tenuipalpidae) em clones de seringueira?
}

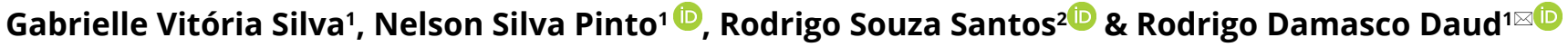

1. Laboratório de Taxonomia, Ecologia e Interações de Aracnídeos (TEIA), Universidade Federal de Goiás, GO, Brasil. 2. Laboratório de Entomologia, Embrapa Acre, Rio Branco, AC, Brasil.

\section{Entomology Beginners, vol. 2: e023 (2021)}

\begin{abstract}
Resumo. No presente estudo foi avaliado o efeito temperatura sobre a dinâmica populacional de Tenuipalpus heveae Baker em cinco clones de seringueira. O estudo foi conduzido de novembro de 2018 a janeiro de 2019 no jardim clonal de seringueiras do campo experimental da Embrapa Acre, Rio Branco, estado do Acre, nos clones CPAA C18, FDR 5788, FX 3899, PB 312 e RRIM 600. Semanalmente foram retiradas, aleatoriamente, oito folhas do terço inferior da copa de quatro plantas de cada clone. Os ácaros coletados foram montados em lâminas de microscopia em meio de Hoyer. A relação entre a temperatura média e a abundância de $T$. heveae foi testada por meio de um modelo linear generalizado com distribuição de Poisson. Foi amostrado um total de 2.909 ácaros em 6.720 folíolos analisados. Observou-se um efeito negativo da temperatura sobre a abundância de T. heveae.
\end{abstract}

Palavras-chave: Acariformes; Ácaro-plano-da-seringueira; Euphorbiaceae; Hevea brasiliensis; Resistência de plantas.

\section{How does temperature affect population dynamics of Tenuipalpus heveae (Acari, Tenuipalpidae) in rubber tree clones?}

Abstract. In the present study, the effect of temperature on the population dynamics of Tenuipalpus heveae Baker in five rubber tree clones was evaluated. The study was conducted from November 2018 to January 2019 in the rubber tree clonal garden of the experimental field of Embrapa Acre, municipality of Rio Branco, state of Acre, Brazil in the clones CPAA C18, FDR 5788, FX 3899 , PB 312 and RRIM 600. Eight leaves were randomly removed weekly from the lower third of the canopy of four plants of each clone. The collected mites were mounted on microscope slides in Hoyer's medium. Thus, the relationship between mean temperature and $T$. heveae abundance was tested using a generalized linear model with Poisson's distribution. A total of 2,909 mites were sampled from 6,720 leaflets analyzed. A negative effect of temperature on $T$. heveae abundance was observed.

Keywords: Acariformes; Rubber tree red flat mite; Euphorbiaceae; Hevea brasiliensis; Plant resistance.

A seringueira, Hevea brasiliensis (Willd. ex Adr. de Juss.) Müell. Arg. (Euphorbiaceae), é uma planta nativa da região amazônica, considerada a principal fonte comercial de borracha natural no mundo (PEREIRA et al., 2000). Devido à sua importância econômica, essa planta foi introduzida em várias regiões do Brasil cultivada em sistema de monocultura. Porém, a expansão da área plantada de seringueira, associada às diversas condições edafoclimáticas dessas regiões, favoreceu a ocorrência de várias espécies de ácaros e insetos fitófagos que, dependendo de seus níveis populacionais, se tornaram um problema para a cultura, interferindo na fisiologia da planta e, consequentemente, na produção de borracha (PONTIER et al., 2000; FERES et al., 2010).

Os ácaros (Acari: Arachnida) são artrópodes diminutos e muito abundantes no ambiente, podendo ser encontrados nos mais diversos micro-habitats, inclusive em plantas nativas e cultivadas (MORAES e FLECHTMANN, 2008). Dentre as espécies de ácaros plantícolas associados a $H$. brasiliensis destacam-

\section{Editado por:}

Noeli Juarez Ferla

\section{Histórico Editoral:}

Recebido em: 07.10.2021

Aceito em: 09.12.2021

Publicado em: 14.12.2021 se Calacarus heveae Feres (Eriophyidae) e Tenuipalpus heveae Baker (Tenuipalpidae), considerados pragas-chave da cultura no Brasil (VIEIRA e GOMES, 2003).

Tenuipalpus heveae pode causar danos significativos às plantas de seringueira quando ocorre em alta densidade populacional (VIEIRA et al., 2009). As folhas atacadas tornam-se amareladas e posteriormente caem, diminuindo a capacidade fotossintética das plantas infestadas (VIEIRA et al., 2009). No Brasil, essa espécie já foi registrada em seringais nos estados do Acre, Amapá, Pará, Amazonas, Bahia, Mato Grosso e São Paulo (BAKER, 1945; FERES et al., 2002; DAUD e FERES, 2007; DEUS et al. 2012; CASTRO et al., 2018; SANTOS et al., 2020).

As variáveis climáticas podem afetar a abundância e a distribuição de ácaros e, por conseguinte, afetar a susceptibilidade do cultivo ao ataque de espécies fitófagas. A temperatura e a pluviosidade podem interferir negativa ou positivamente na dinâmica populacional e estão diretamente correlacionadas ao aumento ou diminuição da população

\section{Autor Correspondente:}

Rodrigo Damasco Daud rodrigodaud@ufg.br

Agência(s) de Fomentos:

Conselho Nacional de Desenvolvimento Científico e Tecnológico 
de insetos fitófagos em suas plantas hospedeiras (AWMACK e LEATHER, 2002). Ainda, podem resultar na variação da dominância e abundância de artrópodes fitófagos em uma mesma cultura quando plantada em diferentes localidades (LARA, 1991; GONÇALVES et al., 1999).

Devido à importância de $T$. heveae para a heveicultura nacional é necessário conhecer o efeito das condições climáticas na ocorrência e abundância dessa espécie em diferentes regiões, a fim de possibilitar um plano eficiente de seu manejo no campo e prever surtos populacionais dessa espécie. Neste sentido, o objetivo desse estudo foi avaliar o efeito de variáveis climáticas sobre a abundância e dinâmica populacional de $T$. heveae em cinco clones de seringueira no estado do Acre. Os dados analisados são complementares aos resultados obtidos por Santos et al. (2020), os quais fizeram o primeiro registro de $T$. heveae no estado do Acre e, cuja pesquisa na mesma área experimental, embasou o presente trabalho.

O estudo foi realizado no jardim clonal de seringueiras, localizado no campo experimental da Embrapa Acre, Rio Branco, AC (0957'48,3" S e 68 $05^{\circ} 55,4^{\prime \prime}$ O), no período de novembro de 2018 a janeiro de 2019. O clima da região é quente e úmido e a temperatura média anual é de $26{ }^{\circ} \mathrm{C}$, com precipitação média anual em torno de $2.000 \mathrm{~mm}$ (ACRE, 2010).

As plantas avaliadas estão dispostas no jardim clonal, em espaçamento de $1 \mathrm{~m} \times 1 \mathrm{~m}$, não sofreram aplicação de produtos fitossanitários durante o período do estudo e foram cultivadas seguindo os tratos culturais recomendados para a cultura.

Semanalmente, foram coletadas folhas de plantas dos clones CPAA C18, FDR 5788, FX 3899, PB 312 e RRIM 600. Em cada coleta foram amostradas aleatoriamente oito folhas do terço inferior da copa em quatro plantas de cada clone, totalizando 32 folhas por clone/coleta (SANTOS et al., 2020).

Após a coleta, as folhas foram acondicionadas em sacos de papel identificados e levadas ao Laboratório de Entomologia da Embrapa Acre, onde permaneciam em câmara climatizada à 20 $\pm 1^{\circ} \mathrm{C}$ até o momento da análise. Em seguida, as faces abaxial e adaxial de cada folíolo eram observadas sob microscópio estereoscópio, sendo todos os ácaros capturados com auxílio de um pincel e montados em lâminas de microscopia em meio de Hoyer (MORAES e FLECHTMANN, 2008). Ácaros de outras espécies não foram considerados nesta pesquisa e $T$. heveae foi escolhido pela sua importância econômica e, por ser o ácaro mais abundante no levantamento realizado por Santos et al. (2020) nesse jardim clonal.

Após preparadas, as lâminas foram enviadas ao especialista reportado nos agradecimentos para a identificação. Durante o período de estudo, a pluviosidade semanal acumulada $(\mathrm{mm})$ e a temperatura média semanal foram registradas continuamente por uma estação meteorológica portátil, instalada no campo experimental da Embrapa Acre.

Antes de realizar as análises, foi avaliada a correlação entre a pluviosidade semanal acumulada e a temperatura média semanal. Em razão da correlação observada entre essas duas variáveis $(R=-0,33 ; t=-2,86, d f=68, p$-value $=0,006)$, após a análise de resíduos, optou-se por manter apenas a variável temperatura na análise, uma vez que essa variável apresentou melhor ajuste no modelo. Foi utilizada um modelo linear generalizado misto ( $\mathrm{glmm}$ ) com distribuição de Poisson, utilizando o software estatístico R (R CORE TEAM, 2021). No glmm usamos a temperatura média como variável preditora fixa, o clone de seringueira como variável preditora aleatória e a abundância de $T$. heveae como a variável resposta. A escolha pela distribuição de Poisson deve-se à natureza da variável resposta, uma vez que se tratam de dados de contagem. As análises foram realizadas usando o software $\mathrm{R}$, por meio do pacote Ime4 (BATES et al., 2015).

Foram coletados um total de 2.909 espécimes de $T$. heveae (imaturos e adultos). No clone FX 3899 foram registrados 1.156 ácaros, 853 em FDR 5788, 424 em PB 312, 415 em RRIM 600 e 61 ácaros no clone CPPA C18. Em relação à dinâmica populacional de $T$. heveae, a variação entre os clones foi perceptível, sendo FX 3899 o mais infestado, com 39,7\% dos ácaros, enquanto CPPA C18 foi o clone menos infestado, com 2,1\%.

A temperatura média foi de $25,7 \pm 0,51^{\circ} \mathrm{C}$ e a precipitação média foi de 43,2 $\pm 18,18 \mathrm{~mm}$. Foi verificado um efeito negativo da temperatura sobre a abundância de $T$. heveae (Tabela 1). Foi observada diminuição média de 2,1 indivíduos de $T$. heveae a cada aumento de $1{ }^{\circ} \mathrm{C}$ na temperatura para os diferentes clones (Figura 1).

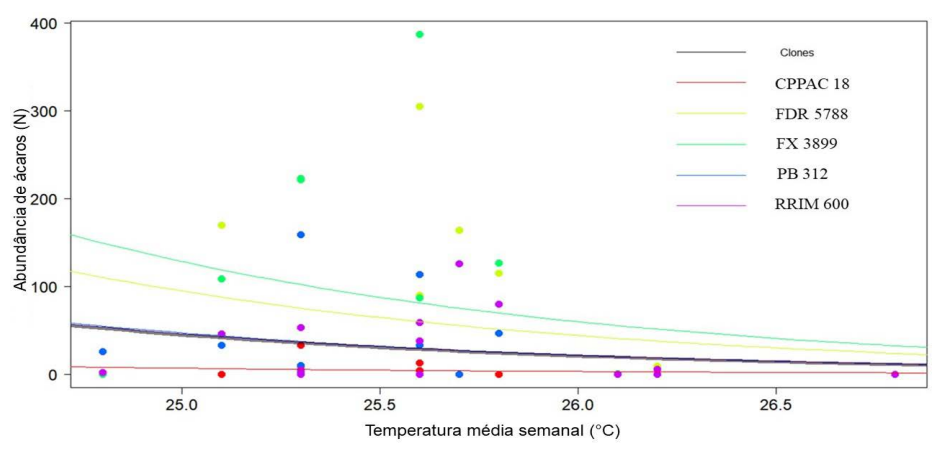

Figura 1. Relação entre o número de indivíduos de Tenuipalpus heveae em cinco clones de seringueira com a temperatura média semanal, em Rio Branco, AC.

Tabela 1. Relação entre a abundância de Tenuipalpus heveae e a temperatura média semanal $\left({ }^{\circ} \mathrm{C}\right)$ observada no glmm e a distribuição de Poisson. Valores em negrito indicam uma relação significativa ao nível de $5 \%$ de probabilidade (alfa $<0,05$ ). $O$ valor de $\beta$ corresponde ao tamanho de efeito da temperatura sobre a abundância de ácaros. O valor Z corresponde à estatística do teste $\mathrm{glmm}$ com distribuição de Poisson e $\mathrm{P}$ corresponde à estimativa do valor da probabilidade.

\begin{tabular}{ccccc}
\hline & $\begin{array}{c}\text { Inclinação da } \\
\text { reta }(\boldsymbol{\beta})\end{array}$ & $\begin{array}{c}\text { Erro } \\
\text { Padrão }\end{array}$ & $\mathbf{Z}$ & $\mathbf{P}$ \\
\hline Intercepto & 22,868 & 10,459 & 2,19 & 0,029 \\
Temperatura & $\mathbf{- 2 , 1 4 4}$ & $\mathbf{1 , 4 5 4}$ & $\mathbf{- 2 , 0 3}$ & $\mathbf{0 , 0 4 2}$ \\
\hline
\end{tabular}

Os resultados do presente trabalho indicam que a temperatura apresenta um efeito negativo na abundância de $T$. heveae nos clones de seringueira avaliados. Esses resultados sugerem que a temperatura pode agir como filtro ambiental na regulação das populações em condições de campo. Ademais, além das variáveis climáticas, as diferenças nos resultados da dinâmica populacional entre os clones podem estar relacionadas com as condições fisiológicas e as características químicas e/ou físicas de cada clone, as quais, também podem influenciar na abundância dos ácaros em seringueiras (DAUD e FERES, 2007).

Entre os principais fatores climáticos favoráveis ao desenvolvimento de ácaros estão os períodos de estiagem, com baixa umidade relativa do ar, aliada à temperatura em torno de $25^{\circ} \mathrm{C}$ (ANDRADE et al., 2008). Dessa forma, o padrão de dinâmica populacional do ácaro observado neste estudo corrobora com essa predição.

Diferindo dos resultados observados neste estudo, nos estados de São Paulo, Mato Grosso e Bahia, o pico de abundância de $T$. heveae ocorre de janeiro a abril (FERES et al., 2002; HERNANDES e FERES, 2006; DAUD e FERES, 2007; CASTRO et al., 2018). No presente estudo, a menor abundância de $T$. heveae foi registrada no mês de janeiro. A temperatura é um dos principais fatores que afetam o desenvolvimento populacional dos ácaros, sendo que as temperaturas mais quentes usualmente aceleram a velocidade de reprodução na maioria das espécies, enquanto as temperaturas baixas ou mudanças bruscas na temperatura retardam o ciclo biológico, reduzindo o tamanho populacional (ROGGIA, 2010; MORAES e FLECHTMANN, 2008). Entretanto, 
em nosso estudo, verificamos que o aumento de temperatura a partir dos $25^{\circ} \mathrm{C}$ afeta negativamente o crescimento populacional de T. heveae em clones de seringueira. É possível que isso se deva a grandes variações de temperatura, como observadas ao longo desse estudo $\left(24^{\circ} \mathrm{C}\right.$ a $27^{\circ} \mathrm{C}$, uma variação de $3^{\circ} \mathrm{C}$ em média). Recentemente foi demonstrado que existem diferenças sobre o desenvolvimento e, consequentemente, sobre a estrutura da população, em ambientes com temperaturas constantes quando comparados com ambientes com temperaturas flutuantes, com efeitos negativos em ambientes com flutuações e temperaturas mais altas (RISMAYANI et al., 2021).

Existem relatos sobre a influência direta do aumento da temperatura e precipitação na redução de populações de ácaros (PEDRO NETO, 2009; ROGGIA, 2010). A precipitação pode interferir na redução das populações de ácaros, pois chuvas fortes causam aumento da umidade relativa, além da possibilidade da lavagem das folhas, causando a remoção mecânica dos ácaros em suas superfícies (PEDRO NETO, 2009). Além do mais, a alta pluviosidade favorece epizootias do fungo patogênico Hirsutella thompsonii Fisher, que atua como agente de controle biológico de ácaros fitófagos em cultivos de seringueira, incluindo T. heveae (DEMITE e FERES, 2008).

Neste estudo foi observado um efeito negativo da temperatura sobre a abundância de $T$. heveae, com as maiores abundâncias em temperaturas mais amenas. Esse resultado corrobora com os resultados de Reis et al. (2000) para outros táxons de ácaros que ocorrem em citros. Essa diminuição pode estar relacionada com efeitos da temperatura sobre o ciclo de vida, diminuindo o recrutamento (CHIAVEGATO, 1986). Assim, quanto menor a temperatura, melhores serão as condições para o crescimento da população desse ácaro. No entanto, estudos mais extensivos e prolongados sobre a dinâmica populacional de $T$. heveae são fortemente recomendados, a fim de determinar o período de ocorrência, picos populacionais e subsidiar práticas racionais e eficientes de manejo em seringais de cultivo no estado do Acre.

\section{AGRADECIMENTOS}

Os autores agradecem ao Dr. Noeli Juarez Ferla (Universidade do Vale do Taquari - UNIVATES, Lajeado, RS) pela identificação dos ácaros amostrados no estudo e aos dois revisores anônimos pelas valiosas críticas e sugestões ao trabalho. NSP agradece ao CNPq a bolsa de pós-doutorado concedida (160656/2019-2).

\section{REFERÊNCIAS}

ACRE. Governo do Estado do Acre. Zoneamento ecológicoeconômico do Estado do Acre, Fase II (Escala 1: 250.000): Documento síntese. $2^{\text {a }}$ ed. Rio Branco: SEMA, 2010. 356p.

ANDRADE, D. J.; OLIVEIRA, C. A. L.; FALCONI, R. S.; PATTARO, F. C.; FERNANDES, E. J. Efeito do déficit hídrico e da presença do vírus da Leprose dos citros na temperatura foliar e sua relação com a população de Brevipalpus phoenicis e a severidade da doença em plantas cítricas. Laranja, v. 29. n. 1-2, p. 1-15, 2008.

AWMACK, C. S.; LEATHER, S. R. Host plant quality and fecundity in herbivorous insects. Annual Review of Entomology, v. 47, p. 817-844, 2002. DOI: https://doi.org/10.1146/annurev. ento.47.091201.145300

BATES, D.; MÄCHLER, M.; BOLKER, B.; WALKER, S. Fitting linear mixed-effects models using Ime4. Journal of Statistical Software, v. 67, n. 1, p.1-48, 2015. DOI: https://doi.org/10.18637/jss.v067. i01

BAKER, E. W. Mites of the genus Tenuipalpus (Acarina:
Trichadenidae). Proceedings of the Entomological Society of Washington, v. 47, n. 2, p. 33-38, 1945.

CASTRO, E. B.; NUVOLONI, F. M.; FERES, R. J. F. Population dynamics of the main phytophagous mites associated with rubber tree plantations in the state of Bahia, Brazil. Systematics and Applied Acarology, v. 23, n. 8, p. 1578-1591, 2018. DOI: https://doi.org/10.11158/saa.23.8.8

CHIAVEGATO, L. G. Biologia do ácaro Brevipalpus phoenicis em citros. Pesquisa Agropecuária Brasileira, v. 21, n. 8, p. 813816, 1986.

DAUD, R. D.; FERES, R. J. F. Dinâmica populacional de ácaros fitófagos (Acari, Eriophyidae, Tenuipalpidae) em seis clones de seringueira no sul do Estado de Mato Grosso. Revista Brasileira de Entomologia, v. 51, n. 3, p. 377-381, 2007. DOI: https://doi.org/10.1590/S0085-56262007000300016

DEMITE, P. R.; FERES, R. J. F. Seasonality of pathogenic fungi in mites of rubber tree plantations adjacent to fragments of Cerrado. Brazilian Journal of Biology, v. 68, n. 3, p. 535-538, 2008. DOI: https://doi.org/10.1590/S1519-69842008000300010

DEUS, E. G.; SOUZA, M. S. M.; MINEIRO, J. L. C.; ADAIME, R.; SANTOS, R. S. Mites (Arachnida: Acari) collected on rubber trees Hevea brasiliensis (Willd. ex A. Juss.) Müll. Arg. in Santana, Amapá state, Brazil. Brazilian Journal of Biology, v. 72, n. 4, p. 915-918, 2012. DOI: https://doi.org/10.1590/S1519-69842012000500019

FERES, R. J. F.; D. de C. ROSSA-FERES; DAUD, R. D.; SANTOS, R. $\mathrm{S}$. Diversidade de ácaros em seringueiras (Hevea brasiliensis Müell. Arg., Euphorbiaceae) na região noroeste do estado de São Paulo, Brasil. Revista Brasileira de Zoologia, v. 19, n. 1, p.137-144, 2002.

FERES, R. J. F.; DEL'ARCO, M.; DAUD, R. D. Biological cycle of Tenuipalpus heveae Baker (Acari, Tenuipalpidae) on leaflets of three rubber tree clones. Revista Brasileira de Entomologia, v. 54, n.2, p. 298-303, 2010. DOI: https://doi.org/10.1590/S008556262010000200013

GONÇALVES, P. S.; BORTOLETTO, N.; ORTOLANI, A. A.; BELLETTI, G. O.; SANTOS, W. R. Desempenho de novos clones de seringueira. III. Seleções promissoras para a região de Votuporanga, Estado de São Paulo. Pesquisa Agropecuária Brasileira, v. 34, n. 6, p. 971-980, 1999. DOI: https://doi.org/10.1590/S0100-204X1999000600007

HERNANDES, F. A.; FERES, R. J. F. Diversidade e sazonalidade de ácaros (Acari) em seringal (Hevea brasiliensis, Müell. Arg.) na Região Noroeste do estado de São Paulo, Brasil. Neotropical Entomology, v. 35, n. 4, p. 523-535, 2006. DOI: https://doi.org/10.1590/S1519-566X2006000400016

LARA, F. M. Fatores que influenciam a manifestação da resistência. In: LARA, F. M. (Ed.). Princípios de resistência de plantas a insetos. São Paulo: Ícone Editora, 1991. 388p.

MORAES, G. J.; FLECHTMANN, C. H. W. Manual de Acarologia: Acarologia básica e ácaros de plantas cultivadas no Brasil. 
Ribeirão Preto: Holos Editora, 2008.

PEDRO NETO, M. Influência de cobertura vegetal do solo e da precipitação pluvial na população de ácaros-praga e de ácaros predadores em cafeeiro orgânico e convencional. Tese (Doutorado). Universidade Federal de Lavras, Lavras, MG, 2009.

PEREIRA, J. P.; DORETTO, M.; LEAL, A. C.; CASTRO, A. M. G. de; RUCKER, N. A. Cadeia produtiva da borracha natural. Análise diagnóstica e demandas atuais do Paraná. Londrina: IAPAR (Documento, 23), 2000. 85p.

REIS, P. R.; SOUZA, J. C.; SOUSA, E. O.; TEODORO A. V. Distribuição espacial do ácaro Brevipalpus phoenicis (Geijskes) (Acari: Tenuipalpidae) em cafeeiro (Coffea arabica L.). Anais da Sociedade Entomológica do Brasil, v. 29, n. 1, p. 177-183, 2000.

PONTIER, K. J. B.; MORAES, G. J. de; KREITER, S. Biology of Tenuipalpus heveae (Acari, Tenuipalpidae) on rubber tree leaves. Acarologia, v. 41, n. 4, p. 423-427, 2000.

ROGGIA, S. Caracterização de fatores determinantes dos aumentos populacionais de ácaros tetraniquídeos em soja. 2010. 154 f. Tese (Doutorado) - Escola Superior de Agricultura "Luiz de Queiroz", Universidade de São Paulo, Piracicaba, SP.

R CORE TEAM. R A language and environment for statistical computing, 2021. Vienna: The R Foundation for Statistical Computing, 2018. Disponível em: <https://www.r-project.org/>
Acesso em: 04 dez. 2021.

RISMAYANI, M. S. U; HSIN, C.; TETSUO G. Impact of Constant and FluctuatingTemperatures on Population Characteristics of Tetranychus pacificus (Acari: Tetranychidae). Journal of Economic Entomology, V. 114, n. 2, P. 638-651, 2021. DOI: https://doi.org/10.1093/jee/toaa327

SANTOS, R. S.; FERLA, N. J.; SCHÜSSLER, M. Primeiro registro e dinâmica populacional do ácaro-plano-vermelho (Acari: Tenuipalpidae) em seringueira no Estado do Acre. In: SEMINÁRIO DA EMBRAPA ACRE DE INICIAÇÃO CIENTÍFICA E PÓS-GRADUAÇÃO, 2. Anais... Rio Branco, AC: Embrapa Acre, 2020. p. 123-128.

VIEIRA, M. R., GOMES, E. C. Ácaros da seringueira: sintomas e controle. In: GONÇALVES, P. S.; BENESI, J. F. C. (Eds.). In: CICLO DE PALESTRAS SOBREA A HEVEICULTURA PAULISTA, 3. Anais... São José do Rio Preto, SP: Secretaria da Agricultura e Abastecimento do Estado de São Paulo e Associação Paulista dos Produtores e Beneficiadores de Borracha, 2003. p. 72-63

VIEIRA, M. R.; SILVA, H. A. de S. da; CARDOSO, M. M. Progênies de seringueira com potencial para conferir resistência a ácaros (Calacarus heveae Feres e Tenuipalpus heveae Baker). Ciência Rural, v. 39, n. 7, p. 1953-1959, 2009. DOI: https://doi.org/10.1590/S0103-84782009005000164 